\title{
Proximate pathways underlying social behavior
}

Dustin R. Rubenstein ${ }^{1,2^{*}}$ and Hans A. Hofmann ${ }^{3,4^{*}}$

${ }^{1}$ Department of Ecology, Evolution and Environmental Biology, ${ }^{2}$ Center for Integrative Animal Behavior, Columbia University, 1200 Amsterdam Avenue, New York, NY 10027, USA

${ }^{3}$ Department of Integrative Biology, ${ }^{4}$ Center for Computational Biology and Bioinformatics, The University of Texas, Austin, Texas 78712, USA

*Corresponding Authors:

dr2497@ columbia.edu, Tel: 212-854-4881

hans@utexas.edu, Tel: 512-524-0675 


\begin{abstract}
The organism is the central unit integrating genomic, developmental, and environmental forces. Animal behavior is fundamental to this process. The integrative study of animal behavior demands numerous interdisciplinary approaches to tackle fundamental questions across multiple levels of organization, diverse taxonomic groups, and various spatial and temporal scales. Social behavior is well-suited for integrative studies since it is ubiquitous, and because sociality represents a pinnacle of biological complexity. We review five proximate pathways that may underlie vertebrate social behavior and its environmental connection: neural circuits, neuroendocrine regulation, gene expression, epigenetic regulation, and genome structure. We suggest researchers take both a "forward" and "reverse" genomics approach, and probe the mechanistic bases of animal social behavior by studying transitions between social states or phenotypes.
\end{abstract}

Keywords: sociality; social phenotype; neural circuit; neuroendocrine; gene expression; epigenetic regulation; genome structure 


\section{Introduction}

All animals integrate information from the external environment with internal physiological information to produce behavior. Although these processes are greatly influenced by selection (natural, sexual, or kin), our understanding of how organisms make behavioral decisions at a mechanistic level and how these "behavioral decision-making mechanisms" evolve is still very limited [1]. Social interactions are central to many of these behavioral decision-making processes, and social behavior in particular is essential to this process because it is the product of reacting to both the social and the ecological environment. Yet, social behaviors are complex and our understanding of their mechanistic bases are often limited compared to other behaviors, particularly in vertebrates where social roles can be more plastic compared to insects. Social behavior research can benefit greatly from an integrative approach examining mechanism and function because social behavior crosses spatial and temporal scales and all levels of biological organization: social behaviors range from pairings that occur only to mate, to interactions over food or other resources, to the formation of complex societies where large groups coordinate their behaviors and exhibit a reproductive division of labor that is either permanent (i.e., caste systems) or temporary (i.e., helper systems) [2].

Behavioral and evolutionary biologists have long recognized the link between genes and behavior, and new technologies have begun to unlock the molecular toolkit underlying complex social behavior [3-5]. Not only have genomic approaches permitted the identification of genes involved in various social and communication behaviors in a range of social species [6], but more detailed studies of the brain in diverse animal lineages have identified brain regions and neuronal populations involved in shaping evolutionarily conserved social behaviors [7-9]. Ultimately, it is necessary to understand (i) the changes in gene expression that occur in response to both external and internal stimuli, (ii) the action of the gene products produced as a result of these molecular changes, and (iii) the neural circuitry (and other physiological processes) in which these changes take place.

The mechanisms underlying social behaviors are often thought of as a "black box" by behavioral ecologists, whereas behavioral neuroscientists rarely think in terms of evolutionary adaptation [2]. Here we focus our review on five non-mutually exclusive proximate pathways that could potentially unlock this black box and underlie the evolution of adaptive social behaviors in a diverse range of vertebrate species: (i) neural circuits; (ii) neuroendocrine regulation; (iii) gene expression; (iv) epigenetic regulation; and (v) genome structure. These pathways link the environment — both social and ecological — to social phenotypes (e.g., mating tactics, breeding roles, dominance rank, etc.) and other complex behaviors through neurons in the brain as well as the genome, its expression, and resulting gene products (Figure 1).

Ultimately, we illustrate a number of relevant proximate pathways underlying social behavior in animals and discuss not only what has been done already, but what is likely to be possible in the future, particularly as we move away from a focus on model organisms and begin to study animals more mechanistically in the wild [10].

\section{Neural circuits}

Neural circuits are more than anatomical structures of connected neurons - they are functional entities that have complex inputs and outputs, regulate their own activity, and ultimately influence behavior. Two neural circuits are thought to be of critical importance for social behavior in mammals: (i) the "social behavior network" consisting of amygdalar and hypothalamic regions that regulate social behavior (e.g., sexual behavior, aggression, parental 
care), which are reciprocally connected and contain sex steroid hormone receptors [7, 11]; and (ii) the "mesolimbic reward system" consisting mostly of telencephalic brain regions and dopaminergic projections from the midbrain ventral tegmental area, which evaluates the salience of an external stimulus [12]. These two neural circuits were present in early vertebrates, and together form a larger "social decision-making network" that regulates adaptive behavior across the five major vertebrate lineages: mammals, birds, reptiles, amphibians, and teleost fishes [8].

\section{Neuroendocrine regulation}

There is increasing evidence that a conserved or deeply homologous molecular toolkit contributes to the regulation of social phenotypes having evolved independently across distantly related species $[9,13]$. In vertebrates, several neuroendocrine and neuromodulatory systems that influence - and are influenced by — social dominance relationships are central to understanding how social behavior is regulated. Three examples of important and well-studied neuroendocrine modulators of social behavior that can act in a causal way to modulate vertebrate social behavior, but are also shaped by environmental and social interactions, are: (i) steroid hormones; (ii) biogenic amines; and (iii) nonapeptides [8]. Steroid hormones and their receptors play a fundamental role in the regulation of social phenotypes, as steroids respond acutely to social stimuli and modulate behavior. For example, the "challenge hypothesis" explains dynamic androgen responses to social challenges [14]. Although the challenge hypothesis was proposed initially for songbirds, it has now been demonstrated across vertebrates [15-17] and in a variety of social contexts [18-20]. Social interactions can also influence the regulation of the hypothalamic-pituitary-adrenal (HPA) axis, which integrates environmental information and the downstream release of glucocorticoids to cause adaptive behavioral responses to external stressors. In many social vertebrates, the social environment (i.e., social status and dominance rank) can also impact HPA function and the release of glucocorticoids [21, 22], which can in turn influence social phenotypes [23]. Similarly, biogenic amines have long been known to influence vertebrate social dominance relationships. The neuromodulator serotonin plays a role in regulating aggressive and defensive behaviors, modulating both baseline activity and the response to challenging stimuli [24]. Finally, nonapeptides and their cognate receptors have also been strongly implicated in the regulation of social dominance behavior (e.g., arginine vasopressin in mammals or arginine vasotocin in non-mammalian vertebrates) [25-27]. Despite the importance of nonapeptides in individual vertebrate species, we lack a uniform theory for how nonapeptides underlie social behavior because patterns across species are complex [28].

Most studies of the neuroendocrine modulation of animal social behavior have focused on specific key modulators or gene products, a method that mirrors classical approaches to studying candidate genes related to behavioral regulation. Yet, just as with the advent of new sequencing technologies that allow us to study all of the genes expressed during a behavioral event, we can also study all of the gene products produced at one time by examining the proteome. Proteomics (i.e., analyses of the complete protein complement expressed by a genome) may now be accessible to the study of animal behavior due to advances in mass spectrometry and bioinformatics, as well as the availability of whole genome sequences for nonmodel organisms $[29,30]$. Although proteomic studies in non-model organisms are in their infancy, work on behavioral plasticity, task specialization, and caste specialization in Hymenoptera [31, 32] demonstrates the potential of this approach for the study of vertebrate social behavior. 


\section{Gene expression}

By examining neural transcriptomes of social species, we have begun to understand the dynamic and flexible nature of genome activity in the brain, and identify gene modules (i.e., sets of coregulated genes or proteins) [33] that are associated with variation in social and reproductive behaviors in diverse species [1, 3, 34]. Eusocial insects have attracted considerable attention with regards to the functional genomics of social behavior, largely because they display highly plastic yet stereotypical transitions between castes and other life history stages (e.g., nurses and workers in honey bees [35]) and because the huge variability in the level of sociality across species make them ideal model systems for comparative studies [6, 36, 37]. Plastic social phenotypes in vertebrates are generally highly dynamic (i.e., change frequently) and thus are somewhat more difficult to study from a genomic perspective compared to eusocial insects. However, much progress has been made, and numerous general insights have emerged. For example, we now know that the expression of the genome can change much more rapidly and dramatically in response to environmental stimuli than anyone thought possible (e.g., $\sim 10 \%$ of protein coding genes expressed in only $30 \mathrm{~min}$ [38-40]). These dynamic properties likely reflect real-time adjustments in the activity of gene networks in response to - and in preparation for - changes in the activity of both neural circuits and neuroendocrine modulation [41]. Moreover, a large fraction of the genome can be involved in these responses rather than just a few genes [35, 42]. For example, plastic behavioral phenotypes appear to be regulated by particular functional groups or genes $[43,44]$ and it appears that a small set of transcription factors governs global changes in response to different environmental or social stimuli, giving rise to co-regulated gene sets or modules $[45,46]$.

Importantly, gene expression profiles can vary considerably across brain regions [47], underscoring the importance of examining individual brain nuclei or even single neurons as opposed to whole brains. In fact, understanding information processing in neural circuits that result in complex behaviors ultimately requires systematic characterization of the participating cell types and their connections, and the ability to measure and perturb their activity $[48,49]$. Finally, the few comparative transcriptomics studies that have been conducted suggest that molecular pathways underlying independently evolved behavioral phenotypes might be conserved across divergent taxa [50-52].

\section{Epigenetic regulation}

Epigenetic mechanisms are an alternative and promising direction for studying the molecular basis of social phenotypes, particularly where social roles are highly plastic. Epigenetic changes involve molecular processes that alter gene expression, including the addition of a methyl group to cytosine DNA bases (termed DNA methylation), post-translational modification to histone proteins that shape the structure of chromatin (termed histone modification), or the regulatory actions of RNA molecules [53]. Work from eusocial insects, which have become models for linking epigenetics to social behavior [54,55], suggests that DNA methylation and histone modification can influence social phenotypes through life phase transitions and caste differentiation [reviewed in 56, 57, 58]. For example, patterns of DNA methylation $[59,60]$ and histone modification [61] vary across castes in eusocial Hymenoptera. Although less work has been done in social vertebrates, levels of DNA methylation in the promoter of the glucocorticoid receptor during early life are predictive of social phenotypes later in life in cooperatively breeding birds [62], and adversity in the social and ecological environment can also influence global patterns of DNA methylation across the genome in free-living primates [63]. Further work 
linking DNA methylation, histone modification, gene expression, and social phenotypes is needed to determine the importance of epigenetic regulation as a potential pathway underlying vertebrate social behavior.

\section{Genome structure}

The approaches discussed above are focused on uncovering the function of specific genes and gene networks that underlie complex behaviors, including those related to social behavior. Although determining the causal mechanisms of social behavior is important [2], we must also consider how social behavior both influences and is influenced by the structure of the genome and the rate at which it evolves. Most of the work examining the relationship between genome structure and social behavior has been done in invertebrates. For example, genome size has been hypothesized to be constrained by sociality [64], but comparative studies in eusocial insects have found limited evidence for the relationship between sociality and genome size $[65,66]$. However, a recent study suggests that eusocial bees have fewer transposable elements than less social and non-social species [67], perhaps explaining why eusocial species in some taxonomic groups have smaller genome sizes. Although recombination rates have also been suggested to play a role in the relationships between genome size and sociality [68], this topic remains woefully understudied. Thus, although determining the genes that underlie sociality and different social phenotypes is important, to truly understand the relationship between the genome and social behavior, we must consider how behavior influences not only gene function, but also the structure of the genome and its rate of evolution.

\section{A path forward: forward/reverse genomics and studying social transitions}

Genomics has clearly transformed our understanding of behavioral plasticity and changed the way we think about social evolution, yet the field has also been criticized for its apparent lack of concrete hypotheses and the uninformative gene lists that often result from these studies [5]. While it is indeed relatively easy to obtain a wealth of transcriptional information, identifying the genes or gene networks that are causal in the behavioral context under study is much more challenging. In the same manner that geneticists advance the field by using reverse genetics [69], it is thus becoming increasingly important that these "forward genomic" studies are followed up with "reverse genomic" analyses that examine the functional causes and consequences of differential gene expression patterns [3]. In other words, once novel candidate genes or pathways have been identified via genomic studies (forward genomics), we must use experimental tests on a genomic scale to further dissect the contribution of each gene to the behavioral phenotype (reverse genomics) [e.g., 70]. Additionally, as the field of animal behavior progresses, we must take a comparative approach to study these mechanisms in (i) closely related species exhibiting different social behaviors, (ii) unrelated species exhibiting similar social behaviors, and (iii) individuals in the same species exhibiting different social phenotypes. Comparative biology has always been a hallmark of animal behavior and evolutionary biology, and it must remain so as the discipline becomes more integrative [71].

Although the tremendous plasticity in vertebrate social phenotypes has made studying the genomics of social behavior challenging, this variation also lends itself to detailed analyses of the neuromolecular mechanisms underlying social behavior using transitions between distinct social phenotypes (e.g., from subordinate to dominant, from helper to breeder, from sneaker to nesting male) that are often experimentally accessible. For example, individuals of group-living species frequently form rank-ordered hierarchies that are generally seen as an emergent property 
of individual behavioral rules such as winner and loser effects (e.g., winners of previous conflicts are more likely to escalate a current conflict, whereas losers of previous conflicts are less likely to do so) $[72,73]$. The resulting social dominance phenotype consists of a range of complex traits that include aggressive displays and associated physiological/morphological traits, territorial defense, and signals communicating social status. As a consequence, hierarchical dominance relationships appear to be remarkably similar across species and depend upon similar regulatory pathways (e.g., steroid hormones, neuropeptides, biogenic amines), even though this kind of social system has clearly evolved independently numerous times in many lineages [72].

\section{Conclusions}

Integrative research in animal behavior can be paradigm-shifting or paradigm-reinforcing, and it is important to strike a critical balance between developing novel ideas and testing existing ones $[71,74]$. The study of social behavior lends itself to integrating across disciplines now more than ever because new tools and techniques are allowing us to unlock the so-called mechanistic "black box" that often paralyzes behavioral ecologists. By focusing efforts to comparatively study five key proximate pathways (neural circuits, neuroendocrine regulation, gene expression, epigenetic regulation, genome structure) underlying a range of social behaviors, we can also overcome the idea of the "phenotypic gambit", which posits that knowledge of mechanism is not required for understanding the function and evolution of behavior $[2,75,76]$. These proximate pathways link the environment - both social and ecological - to the social phenotype (e.g., mating tactics, breeding roles, dominance rank, etc.) and other complex behaviors through neurons in the brain as well as the genome, its expression, and the production of gene products. Importantly, these pathways are not independent, and studying them together will provide a much richer and more holistic understanding of the mechanisms underlying animal social behavior. We therefore recommend two primary approaches to studying the mechanistic bases of social behavior: (i) taking both a "forward" and "reverse" genomics approach to identify relevant genes and examine the functional causes and consequences of their expression patterns; and (ii) examining transitions between different social phenotypes. Ultimately, an integrative understanding of animal social behavior will be important if we wish to gain a deeper understanding of what drives animals to be social, employ the study of social behavior in new biomedical discoveries, or conserve biodiversity through knowledge of how social behaviors allow animals to adapt to climate change.

\section{Acknowledgements}

The participants of the NSF workshop on New Frontiers for the Integrative Study of Animal Behavior helped stimulate many of these ideas. We thank R. Calisi, C. Friesen, and S. GuindreParker for providing comments on previous versions of this manuscript. D.R.R. (IOS-1121435, IOS-1257530, IOS-1439985) and H.A.H. (IOS-0843712, IOS-1354942, IOS 1501704) are supported by the US National Science Foundation. 


\section{References}

*1. O'Connell, LA, HA Hofmann: Genes, hormones, and circuits: an integrative approach to study the evolution of social behavior. Front Neuroendocrinol 2011, 32320-335. This paper lays out a broad framework for studying the evolution of the neural and molecular mechanisms underlying (social) behavior.

*2. Hofmann, H, AK Beery, DT Blumstein, ID Couzin, RL Earley, LD Hayes, PL Hurd, EA Lacey, SM Phelps, NG Solomon et al.: An evolutionary framework for studying mechanisms of social behavior. Trends Ecol Evol 2014, 29581-589.

In this essay, the authors revisit Tinbergen's Four Questions and call for an integrative approach to the study of social behavior.

**3. Harris, RM, HA Hofmann: Neurogenomics of behavioral plasticity. Adv Exp Med Biol 2014, 781149-168.

In this essay, "reverse genomics" is proposed as an approach to move beyond correlations between gene expression and physiology and behavior.

4. Rittschof, CC, GE Robinson: Genomics: moving behavioral ecology beyond the phenotypic gambit. Anim Behav 2014, 92263-270.

5. Calisi, RM, M MacManes: RNAseq-ing a more integrative understanding of animal behavior. Current Opinion in Behavioral Science 2015, 665-68.

6. Toth, AL, JF Tooker, S Radhakrishnan, R Minard, MT Henshaw, CM Grozinger: Shared genes related to aggression, rather than chemical communication, are associated with reproductive dominance in paper wasps (Polistes metricus). BMC Genomics 2014, 1575.

7. Goodson, JL: The vertebrate social behavior network: evolutionary themes and variations. Horm Behav 2005, 4811-22.

8. O'Connell, LA, HA Hofmann: The vertebrate mesolimbic reward system and social behavior network: a comparative synthesis. J Comp Neurol 2011, 5193599-3639.

**9. O’Connell, LA, HA Hofmann: Evolution of a vertebrate social decision-making network. Science 2012, 3361154-1157.

The authors show that the expression patterns of key neuroendocrine and neuromodulatory genes are highly conserved in the vertebrate social decision-making network.

10. Taborsky, M, HA Hofmann, AK Beery, DT Blumstein, LD Hayes, EA Lacey, EP Martins, SM Phelps, NG Solomon, DR Rubenstein: Taxon matters: promoting integrative studies of social behavior. Trends Neurosci 2015, 38189-191.

11. Newman, SW: The medial extended amygdala in male reproductive behavior. A node in the mammalian social behavior network. Ann NY Acad Sci 1999, 877242-257.

12. Wise, RA: Forebrain substrates of reward and motivation. J Comp Neurol 2005, 493115-121.

13. Toth, AL, GE Robinson: Evo-devo and the evolution of social behavior: brain gene expression analyses in social insects. Cold Spring Harbor Symp Quant Biol 2009, 74419426.

14. Wingfield, JC, RE Hegner, AM Dufty, GF Ball: The challenge hypothesis: theoretical implications for patterns of testosterone secretion, mating systems, and breeding strategies. Am Nat 1990, 136829-846.

15. Greenberg, N, D Crews: Endocrine and behavioral responses to aggression and social dominance in the green anole lizard, Anolis carolinensis. Gen Comp Endocrinol 1990, 77246-255. 
16. Emerson, SB, DL Hess: The role of androgens in opportunistic breeding, tropical frogs. Gen Comp Endocrinol 1996, 103220-230.

17. Oliveira, RF, K Hirsschenhauser, LA Carneiro, AVM Canario: Social modulation of androgen levels in male teleost fish. Comp Biochem Physiol B 2002, 132203-215.

18. Goymann, W, MM Landys, JC Wingfield: Distinguishing seasonal androgen responses from male-male androgen responsiveness--revisiting the Challenge Hypothesis. Horm Behav 2007, 51463-476.

19. Gleason, ED, MJ Fuxjager, TO Oyegbile, CA Marler: Testosterone release and social context: when it occurs and why. Front Neuroendocrinol 2009, 30460-469.

20. O'Connell, LA, MM Rigney, DW Dykstra, HA Hofmann: Neuroendocrine mechanisms underlying sensory integration of cocial signals. J Neuroendocrinol 2013, 25644-654.

21. Rubenstein, DR, S-F Shen: Reproductive conflict and the costs of social status in cooperatively breeding vertebrates. Am Nat 2009, 173650-661.

22. Creel, S, B Dantzer, W Goymann, DR Rubenstein: The ecology of stress: effects of the social environment. Funct Ecol 2013, 2766-80.

23. Young, AJ, AA Carlson, SL Monfort, AF Russell, NC Bennett, TH Clutton-Brock: Stress and the suppression of subordinate reproduction in cooperatively breeding meerkats. Proc Natl Acad Sci USA 2006, 10312005-12010.

24. Miczek, KA, S Faccidomo, RMM de Almeida, M Bannai, EW Fish, JF Debold: Escalated aggressive behavior: new pharmacotherapeutic approaches and opportunities. Ann NY Acad Sci 2004, 1036336-355.

25. Goodson, JL, D Kabelik: Dynamic limbic networks and social diversity in vertebrates: from neural context to neuromodulatory patterning. Front Neuroendocrinol 2009, 30429-441.

26. Greenwood, AK, AR Wark, RD Fernald, HA Hofmann: Expression of arginine vasotocin in distinct preoptic regions is associated with dominant and subordinate behaviour in an African cichlid fish. Proc $R$ Soc Lond B 2008, 2752393-2402.

27. Hattori, $T, W$ Wilczynski: Comparison of arginine vasotocin immunoreactivity differences in dominant and subordinate green anole lizards. Physiol Behav 2009, 96104-107.

28. Kelly, AM, JL Goodson: Social functions of individual vasopressin-oxytocin cell groups in vertebrates: what do we really know? Front Neuroendocrinol 2014, 35512-529.

29. Valcu, CM, B Kempenaers: Proteomics in behavioral ecology. Behav Ecol 2015, 261-15.

30. Wan, C, B Borgeson, S Phanse, F Tu, K Drew, G Clark, X Xiong, O Kagan, J Kwan, A Bezginov: Panorama of ancient metazoan macromolecular complexes. Nature 2015, 525339-344.

31. Wolschin, F, GV Amdam: Comparative proteomics reveals characteristics of lifehistory transitions in a social insect. Proteome Science 2007, 510.

32. Li, J, J Wu, DB Rundassa, F Song, A Zheng, Y Fang: Differential protein expression in honeybee (Apis mellifera) larvae: underlying caste differentiation. PLOS ONE 2010, 5e13455.

33. Segal, E, N Friedman, D Koller, A Regev: A module map showing conditional activity of expression modules in cancer. Nat Genet 2004, 361090-1098.

34. Mikheyev, AS, TA Linksvayer: Genes associated with ant social behavior show distinct transcriptional and evolutionary patterns. eLife 2015, $4 \mathrm{e} 04775$.

35. Whitfield, CW, A-M Cziko, GE Robinson: Gene expression profiles in the brain predict 
behavior in individual honey bees. Science 2003, 302296-299.

36. Patalano, S, A Vlasova, C Wyatt, P Ewels, F Camara, PG Ferreira, CL Asher, TP Jurkowski, A Segonds-Pichon, M Bachman: Molecular signatures of plastic phenotypes in two eusocial insect species with simple societies. Proc Natl Acad Sci USA 2015, In press.

37. Toth, AL, K Varala, MT Henshaw, SL Rodriguez-Zas, ME Hudson, GE Robinson: Brain transcriptomic analysis in paper wasps identifies genes associated with behaviour across social insect lineages. Proc $R$ Soc Lond B 2010, 2772139-2148.

38. Cummings, ME, J Larkins-Ford, CRL Reilly, RY Wong, M Ramsey, HA Hofmann: Sexual and social stimuli elicit rapid and contrasting genomic responses. Proc $R$ Soc Lond $B$ 2008, 275393-402.

39. Dong, S, KL Replogle, L Hasadsri, BS Imai, PM Yau, S Rodriguez-Zas, BR Southey, JV Sweedler, DF Clayton: Discrete molecular states in the brain accompany changing responses to a vocal signal. Proc Natl Acad Sci USA 2009, 10611364-11369.

40. Simoes, JM, EN Barata, RM Harris, LA O'Connell, HA Hofmann, RF Oliveira: Social odors conveying dominance and reproductive information induce rapid physiological and neuromolecular changes in a cichlid fish. BMC Genomics 2015, 22114.

41. Hofmann, HA: The neuroendocrine action potential. Horm Behav 2010, 58555-562.

42. Renn, S, N Aubin-Horth, HA Hofmann: Fish \& chips: functional genomics of social plasticity in an African cichlid fish. J Exp Biol 2008, 2113041-3056.

43. Aubin-Horth, N, BH Letcher, HA Hofmann: Gene-expression signatures of Atlantic salmon's plastic life cycle. Gen Comp Endocrinol 2009, 163278-284.

44. Sanogo, YO, M Band, C Blatti, S Sinha, AM Bell: Transcriptional regulation of brain gene expression in response to a territorial intrusion. Proc R Soc Lond B 2012, 2794929-4938.

45. Ament, SA, CA Blatti, C Alaux, MM Wheeler, AL Toth, Y Le Conte, GJ Hunt, E GuzmanNovoa, G Degrandi-Hoffman, JL Uribe-Rubio et al.: New meta-analysis tools reveal common transcriptional regulatory basis for multiple determinants of behavior. Proc Natl Acad Sci USA 2012, 109E1801-1810.

46. Simola, DF, L Wissler, G Donahue, RM Waterhouse, M HelmKampf, J Roux, S Nygaard, KM Glastad, DE Hagen, L Viljakainen: Social insect genomes exhibit dramatic evolution in gene composition and regulation while preserving regulatory features linked to sociality. Genome Res 2013, 231235-1247.

47. Oldham, MC, S Horvath, DH Geschwind: Conservation and evolution of gene coexpression networks in human and chimpanzee brains. Proc Natl Acad Sci USA 2006, 10317973-17978.

48. Luo, L, EM Callaway, K Svoboda: Genetic dissection of neural circuits. Neuron 2008, 57634-660.

49. Kravitz, EA, MDL Fernandez: Aggression in Drosophila. Behav Neurosci 2015, 129549563.

50. Gallant, JR, LL Traeger, JD Volkening, H Moffett, P-H Chen, CD Novina, GN Phillips, R Anand, GB Wells, M Pinch: Genomic basis for the convergent evolution of electric organs. Science 2014, 3441522-1525.

*51. Rittschof, CC, SA Bukhari, LG Sloofman, JM Troy, D Caetano-Annolles, A CaashAhmed, M Kent, X Lu, YO Sanogo, PA Weisner: Neuromolecular responses to social challenge: Common mechanisms across mouse, stickleback fish, and honey bee. Proc 
Natl Acad Sci USA 2014, 11117929-17934.

A first attempt to examine conserved genes and gene networks across a diverse array of animals.

**52. Pfenning, AR, E Hara, O Whitney, MV Rivas, R Wang, PL Roulhac, JT Howard, M Wirthlin, P Lovell, G Ganapathy: Convergent transcriptional specializations in the brains of humans and song-learning birds. Science 2014, 3461256846.

This paper provides the best evidence to date that similar (conserved) gene expression networks can be associated with convergently evolved behavioral phenotypes.

53. Bird, A: Perceptions of epigenetics. Nature 2007, 447396-398.

54. Weiner, SA, AL Toth: Epigenetics in social insects: a new direction for undertanding the evolution of castes. Genet Res Int 2012, 2012609810.

55. Yan, H, DF Simola, R Bonasio, J Liebig, S Berger, D Reinberg: Eusocial insects as emerging models for behavioural epigenetics. Nat Rev Genet 2014, 15677-688.

56. Yan, H, R Bonasio, DF Simola, J Liebig, SL Berger, D Reinberg: DNA methylation in social insects: how epigenetics can control behavior and longevity. Anпи Rev Entomol 2015, 60435-452.

57. Bonasio, $\mathrm{R}$ : The role of chromatin and epigenetics in the polyphenisms of castes. Brief Func Genomics 2014, 13235-245.

58. Ernst, UR, MB Van Hiel, G Depuydt, B Boerjan, A De Loof, L Schoofs: Epigenetics and locust life phase transitions. J Exp Biol 2015, 21888-99.

59. Foret, S, R Kucharski, M Pellegrini, S Feng, SE Jacobsen, GE Robinson, R Maleszkya: DNA methylation dynamics, metabolic fluxes, gene splicing, and alternative phenotypes in honey bees. Proc Natl Acad Sci USA 2012, 1094968-4973.

60. Bonasio, R, Q Li, J Lian, NS Mutti, L Jin, H Zhao, P Zhang, P Wen, H Ziang, Y Ding et al.: Genome-wide and caste-specific DNA methylomes of the ants Camponotus floridanus and Harpegnathos saltator. Curr Biol 2012, 221755-1764.

61. Simola, DF, C Ye, NS Mutti, K Dolezal, R Bonasio, J Liebig, D Reinberg, SJ Berger: A chromatin link to caste identity in the carpenter ant Camponotus floridanus. Genome Res 2013, 23486-496.

62. Rubenstein, DR, H Skolnik, A Berrio, FA Champagne, S Phelps, J Solomon: Sex-specific fitness effects of unpredictable early life conditions are associated with DNA methylation in the avian glucocorticoid receptor. Mol Ecol 2016, In press.

63. Lea, AJ, J Altmann, SC Alberts, J Tung: Resource base influences genome-wide DNA methylation levels in wild baboons (Papio cynocephalus). Mol Ecol 2016, In press.

64. Koshikawa, S, S Miyazaki, R Cornette, R Matsumoto, T Miura: Genome size of termites (Insecta, Dictyoptera, Isoptera) and wood roaches (Insecta, Dictyoptera, Cryptocercidae). Naturwissenschaften 2008, 95859-867.

65. Tsutsui, ND, AV Suarez, JC Spagna, JS Johnston: The evolution of genome size in ants. BMC Evol Biol 2008, 864.

66. Ardila-Garcia, AM, GJ Umphrey, TR Gregory: An expansion of the genome size dataset for the insect order Hymenoptera, with a first test of parasitism and eusociality as possible constraints. Insect Mol Biol 2010, 19337-346.

**67. Kapheim, KM, H Pan, C Li, SL Salzberg, D Puiu, T Magoc, HM Robertson, ME Hudson, A Venkat, BJ Fischman et al.: Genomic signatures of evolutionary transitions from solitary to group living. Science $2015, \mathbf{3 4 8} 1139-1143$.

The first study to compare whole genomes in species that differ in social organization. 
68. Kent, CF, S Minaei, BA Harpur, A Zayed: Recomination is associated with the evolution of genome structure and worker behavior in honey bees. Proc Natl Acad Sci USA 2012, 10918012-18017.

69. Alonso, JM, JR Ecker: Moving forward in reverse: genetic technologies to enable genome-wide phenomic screens in Arabidopsis. Nat Rev Genet 2006, 7524-536.

70. O'Connell, LA, HA Hofmann: Social status predicts how sex steroid receptors regulate complex behavior across levels of biological organization. Endocrinology 2012, 1531341-1351.

**71. Rubenstein, DR, H Hofmann, E Akcay, S Alonzo, E Archie, AK Beery, R CalisiRodriguez, K Carelton, B Chow, J Dubnau et al.: New Frontiers for the Integrative Study of Animal Behavior. 2014,

http://www.nsf.gov/bio/pubs/reports/New_Frontiers_for_the_Integrative_Study_of_Animal _Behavior_workshop_report.pdf

This white paper is the result of an NSF-funded workshop that explored the field of animal behavior from an integrative perspective. It defined the concept and laid out a research and training agenda for the field.

72. van Doorn, GS, G Hengeveld, FJ Weissing: The evolution of social dominance II: multiplayer models. Behaviour 2003, 1401333-1358.

73. Hsu, Y, RL Earley, LL Wolf: Modulation of aggressive behaviour by fighting experience: mechanisms and contest outcome. Biol Rev Camb Philos Soc 2006, 8133-74.

74. Monaghan, P: Behavioral ecology and the successful integration of function and mechanism. Behav Ecol 2014, 251019-1021.

75. Grafen, A: Natural selection, kin selection and group selection. In Behavioural Ecology. 1984:62-84.

76. Springer, SA, BJ Crespi, WJ Swanson: Beyond the phenotypic gambit: molecular behavioural ecology and the evoluton of genetic architecture. Mol Ecol 2011, 2022402257. 
Figure 1. Diagram illustrating five proximate pathways that may underlie social behavior across metazoan animals: neural circuits, neuroendocrine regulation, gene expression, epigenetic regulation, and genome structure. These levels link the environment (social and ecological) to the social phenotype (e.g., mating tactics, breeding roles, dominance rank, etc.) through neurons in the brain as well as the genome, its expression, and the production of gene products. This integrative approach will help move the study of animal behavior beyond the "phenotypic gambit", or the so-called "black-box" that has long been used to describe the function and evolution of social behavior. 


\section{Environment}

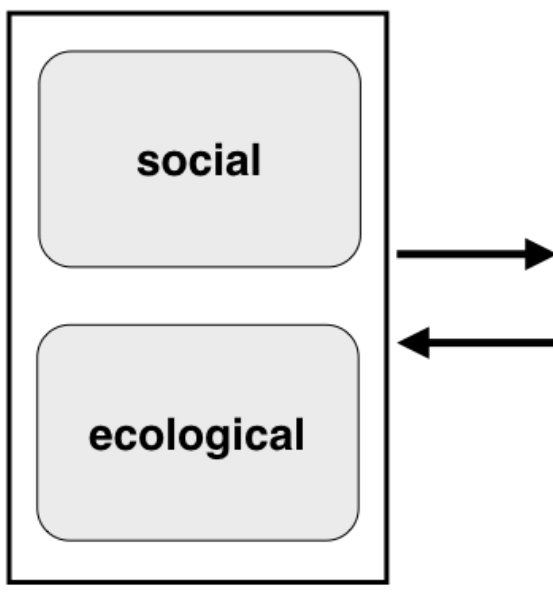

Proximate Pathways

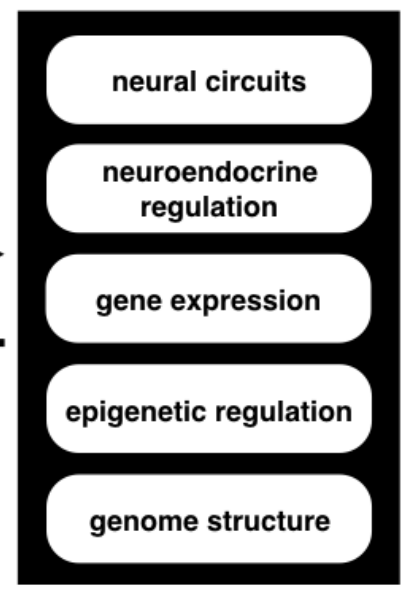

Social Phenotype

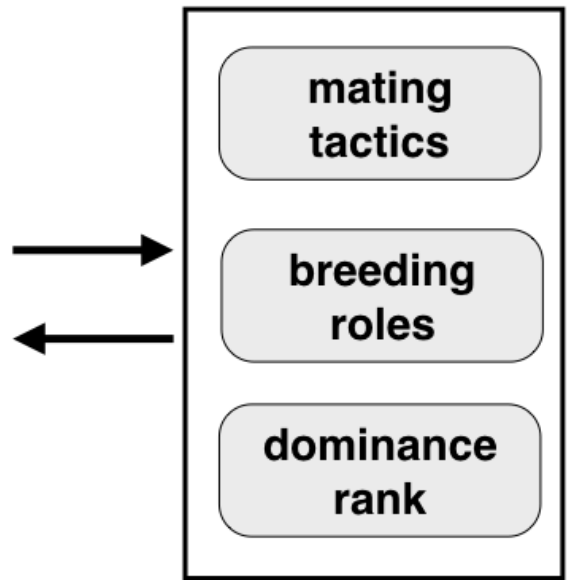

